\title{
Surgical Treatment of Primary Intracardiac Myxoma: 20-Year Experience in "Shahid Modarres Hospital"-A Tertiary University Hospital—Tehran, Iran
}

\author{
Zahra Ansari Aval, ${ }^{1}$ Hamid Ghaderi, ${ }^{2}$ Hassan Tatari, ${ }^{2}$ Mahnoosh Foroughi, ${ }^{2}$ \\ Seyedeh Adeleh Mirjafari, ${ }^{3}$ Mohammad Forozeshfard, ${ }^{4}$ Kamal Fani, ${ }^{5}$ and Isa Khaheshi ${ }^{6}$ \\ ${ }^{1}$ Lung Transplantation Research Center, National Research Institute of Tuberculosis and Lung Diseases (NRITLD), \\ Shahid Beheshti University of Medical Sciences, Tehran, Iran \\ ${ }^{2}$ Cardiovascular Research Center, Department of Cardiovascular Surgery, Shahid Modarres Hospital, \\ Shahid Beheshti University of Medical Sciences, Tehran, Iran \\ ${ }^{3}$ Brain and Spinal Injury Research Center (BASIR), Tehran University of Medical Sciences, Tehran, Iran \\ ${ }^{4}$ Department of Anesthesiology, Semnan University of Medical Sciences, Semnan, Iran \\ ${ }^{5}$ Department of Anesthesiology, Shahid Modarres Hospital, Shahid Beheshti University of Medical Sciences, Tehran, Iran \\ ${ }^{6}$ Cardiovascular Research Center, Shahid Modarres Hospital, Shahid Beheshti University of Medical Sciences, Tehran, Iran \\ Correspondence should be addressed to Hamid Ghaderi; hghaderi@razi.tums.ac.ir
}

Received 3 October 2014; Revised 31 December 2014; Accepted 2 January 2015

Academic Editor: Nejat Akar

Copyright (C) 2015 Zahra Ansari Aval et al. This is an open access article distributed under the Creative Commons Attribution License, which permits unrestricted use, distribution, and reproduction in any medium, provided the original work is properly cited.

Although cardiac tumors are not common they may vary in terms of race and surgical approach in different countries. Method. Patients data of 20 years was collected and evaluated in the "Shahid Modarres Hospital"-a tertiary university hospital-Tehran, Iran. Results. 42 patients with cardiac myxoma (all cases in 20 years) were included in study, 17 males and 25 females, age difference: 13 to 76 years (mean 50.6). Most of patients were in functional classes I, II. 35 patients complained of dyspnea and 3 patients had embolic events. $97.6 \%$ of tumors were primary ( 41 patients) and one tumor was recurrent $(2.4 \%), 85.7 \%$ of tumors (36 cases) were located in LA, and $88.1 \%$ of tumors (37 cases) were pediculated. 40 patients (95\%) had one tumor. In 22 patients (52.3\%) after tumor resection septal defects were repaired primarily while in 18 patients $(42.8 \%)$ the defects were repaired with pericardial patch and In one patient, tumor resected without any septal defect. Mean tumor size was about $5.22 \mathrm{~cm}$ (range of 2.2 to $8.2 \mathrm{~cm}$ ). Postoperatively, 33 patients discharged from hospital without any complication. Discussion. The research reveals that patients' age and gender were similar to that of other studies in other countries while tumor's incidence seems to be higher. 3 patients were diagnosed after remote embolic event and one patient was diagnosed after MI reflecting relatively high tumor complications and late diagnosis. Conclusion. In our study mean time from diagnosis to operation was too long. The patients had more preoperative embolic events and complication. However, size of myxoma and location of that was as same as its rate in the other literature. As recommendation we suggested that in all patients with vague chest pain or remote embolic events cardiac myxomas should be ruled out.

\section{Introduction}

Primary cardiac tumors are rare ones. Their annual incidence is 5 per million among people of 30 to 60 years old [1]. They comprise $0.3 \%$ of all cardiac operations [2]. Although their prevalence is low, they can lead to severe permanent disabilities. They are cause of death if they are diagnosed late or situated in critical locations.

Crafoord reported first cardiac myxoma diagnosis and successful operation by CPB in 1955 [3]. Tumors clinical manifestation and symptom's severity depend on tumor size and its location in heart. 
TABLE 1: Preoperative patients' information from 1994 to 2014 diagnosed with myxoma in Shahid Modarres Hospital.

\begin{tabular}{|c|c|c|}
\hline Presentation (1994-2014) & Parameter & $\%$ \\
\hline \multirow{2}{*}{ Gender } & Male & $17(40.4 \%)$ \\
\hline & Female & $25(59.6 \%)$ \\
\hline \multirow{2}{*}{ Mean age } & & $50.6 \pm 17$ \\
\hline & Range & $13-76$ \\
\hline \multirow{7}{*}{ Age } & $10-19$ years & $1(2.4 \%)$ \\
\hline & $20-29$ years & $3(7.1 \%)$ \\
\hline & $30-39$ years & $5(11.9 \%)$ \\
\hline & $40-49$ years & $6(14.2 \%)$ \\
\hline & $50-59$ years & $15(35.7 \%)$ \\
\hline & $60-69$ years & $9(21.6 \%)$ \\
\hline & $70-80$ years & $3(7.1 \%)$ \\
\hline \multirow{5}{*}{ Diagnostic method } & TTE + TEE + Angio & $4(9.5 \%)$ \\
\hline & TTE + Angio & $14(33.3 \%)$ \\
\hline & TTE + TEE & $1(2.4 \%)$ \\
\hline & TTE & $22(52.4 \%)$ \\
\hline & $\mathrm{TTE}+$ Angio $+\mathrm{CT}$ & $1(2.4 \%)$ \\
\hline \multirow{5}{*}{ Functional class } & Functional class I & $6(14.2 \%)$ \\
\hline & Functional class II & $17(40.6 \%)$ \\
\hline & Functional class III & $14(33.3 \%)$ \\
\hline & Functional class IV & $4(9.5 \%)$ \\
\hline & Unknown & $1(2.4 \%)$ \\
\hline
\end{tabular}

TTE: transthoracic echocardiography; TEE: transoesophageal echocardiography; Angio: angiography; CT: computed tomography.

\section{Method}

This study is descriptive and has been conducted by collecting data of patients admitted in Tehran's Shahid Modarres Hospital-a tertiary university hospital-with diagnosis of cardiac myxoma from 1994 to 2014. Patients' data then got categorized according to their age, gender, clinical manifestations, diagnostic modalities, preoperative functional class, preoperative and postoperative arrhythmia, serologic abnormalities, waiting time to operation, hospital stay, operative findings, concurrent operations, complications, and patients' outcome.

\section{Results}

44 patients with cardiac myxoma underwent operation in 20 years (total cardiac tumor candidates for this study were 52 from which 44 patients had cardiac myxoma and other 8 had other cardiac tumors). Two patients were excluded from study due to lack of data and finally 42 cases were included in the study. 17 (40.4\%) and 25 (59.6\%) were male and female cases, respectively, and patients' mean age was $50.6 \pm 17$ (rang of 13 to 76 ) (Table 1 ).

Mean time from diagnosis to operation was 5.8 days (range from 1 to 48 days) and mean postoperative hospital stay was 12.7 days (range 5 to 32 days). Patients' most common complaints were dyspnea in 23 patients (54.8\%), dyspnea with palpitation in 12 cases (28.5\%), palpitation in 5 patients (11.9\%), weight loss in 9 patients $(21.4 \%)$, and 3 patients admitted in hospital with embolic events (Table 2).
TABLE 2: Clinical presentation (signs and symptoms) of patents diagnosed with myxoma from 1994 to 2014 in Shahid Modarres Hospital.

\begin{tabular}{lc}
\hline Clinical presentation (1994-2014) & $\%$ \\
\hline Chest pain & $3(7.1 \%)$ \\
Dyspnea & $23(54.8 \%)$ \\
Dyspnea with fever and weight loss & $1(2.4 \%)$ \\
Dyspnea with palpitation & $12(28.5 \%)$ \\
CVA with hemiplegia & $1(2.4 \%)$ \\
TIA with IHD & $1(2.4 \%)$ \\
TIA with hemiparesia & $1(2.4 \%)$ \\
Fatigue & $10(23.8 \%)$ \\
Acute myocardial ischemia & $1(2.4 \%)$ \\
Palpitation & $5(11.9 \%)$ \\
Syncope & $1(2.4 \%)$ \\
Weight loss & $9(21.4 \%)$ \\
Peripheral edema & $2(4.8 \%)$ \\
Arthralgia & $1(2.4 \%)$ \\
Incidentally & $1(2.4 \%)$ \\
Unknown & $1(2.4 \%)$ \\
\hline
\end{tabular}

TIA: transient ischemic attack; CVA: cerebrovascular accident; IHD: ischemic heart disease.

Six patients were in NYHA class I (14.3\%), 17 patients in class II (40.5\%), 14 patients in class III (33.3\%), and 4 patients in NYHA class IV (9.5\%). Three patients had a history of cerebral emboli (CVA or TIA) (Table 3). 
TABLE 3: Specification of operation procedures performed for patents diagnosed with myxoma from 1994 to 2014 in Shahid Modarres Hospital.

\begin{tabular}{|c|c|c|}
\hline Operating information & \multicolumn{2}{|c|}{ 1994-2014 } \\
\hline \multirow{4}{*}{ Location } & Left atrium & $36(85.6 \%)$ \\
\hline & Right atrium & $3(7.2 \%)$ \\
\hline & Left and right atrium & $2(4.8 \%)$ \\
\hline & Right ventricle & $1(2.4 \%)$ \\
\hline \multirow{2}{*}{ Kind of tumor } & Primary & $41(97.6 \%)$ \\
\hline & Recurrent & $1(2.4 \%)$ \\
\hline \multirow{3}{*}{ Pediculate or sessile } & Pediculate & $37(90 \%)$ \\
\hline & Sessile & $3(7.2 \%)$ \\
\hline & Unknown & $2(4.8 \%)$ \\
\hline \multirow{4}{*}{ Operating technique } & Primary repair & $22(52.4 \%)$ \\
\hline & Repair with pericardial patch & $18(42.8 \%)$ \\
\hline & Myomectomy & $1(2.4 \%)$ \\
\hline & Unknown & $1(2.4 \%)$ \\
\hline \multirow{4}{*}{ Combination operation } & CABG & $1(2.4 \%)$ \\
\hline & Repair of pulmonary valve & $1(2.4 \%)$ \\
\hline & Mitral valve replacement & $2(4.8 \%)$ \\
\hline & Mitral annuloplasty & $2(4.8 \%)$ \\
\hline Tumor size & \multicolumn{2}{|c|}{$5.22 \pm 1.6 \mathrm{~cm}$, range: 2.2 to $8.2 \mathrm{~cm}$} \\
\hline $\mathrm{CPB}$ time & \multicolumn{2}{|c|}{$85.4 \pm 31 \mathrm{~min}$} \\
\hline $\mathrm{XC}$ time & \multicolumn{2}{|c|}{$55.6 \pm 30 \mathrm{~min}$} \\
\hline
\end{tabular}

CABG: coronary artery bypass graft, CPB: cardiopulmonary bypass, XC: aortic cross clamp.

Transthoracic echocardiography (TTE) was the primary diagnostic modality in all patients; indeed in 22 patients (52.4\%) TTE was the only diagnostic tool but in 5 patients (11.9\%) transoesophageal echocardiography (TEE) was used as well. In 19 patients (45.23\%) coronary angiography was used to rule out concurrent coronary artery disease (Table 1). In addition to echocardiography and angiography, CT-scan was used in one patient.

Laboratory findings were charted as mean WBC: $8294 \pm$ 280 (normal range: 4300-12900), mean HCT: $38.56 \pm 5.38 \%$ (normal range; 26\%-47\%); ESR and CRP were also checked; however, in some patients these data had not been checked, so to analyze these data was out of value.

Almost all tumors were primary (41 patients, 97.6\%) compared to one recurrent case (2.4\%) (Table 3).

Tumors were primary in 41 patients $(97.6 \%)$ and recurrent in one patient $(2.4 \%)$ (Table 3$)$. Tumors' location was in left atrium (LA) in 36 cases $(85.6 \%)$, both atria in 2 cases $(4.8 \%)$, and right atrium (RA) in 3 patients $(7.2 \%)$ and right ventricle (RV) in one patient $(2.4 \%)$. Tumors were pediculated in 37 patients (90\%) and sessile in 3 patients (7.2\%). Tumors were single in 40 patients (95.2\%) and double in one case.

After tumor removal, septal defect was repaired primarily in 22 cases $(52.4 \%)$ while for 18 cases $(42.8 \%)$ pericardial patch was applied. In one patient, tumor was resected without any residual defect in septum. Also, for one case tumor had been located in right ventricular outflow tract with extension into pulmonary valve leaflets for which in addition to the tumor right ventricular septal partially resected and pulmonary valve was repaired.

Along with tumor removal mitral valve replacement was performed in two patients (in one case due to rheumatic mitral stenosis and in one case due to severe mitral regurgitation). Mitral annuloplasty was done in two patients as well (due to severe MR) and one patient underwent CABG concurrent with tumor resection.

Patients were followed up for a mean time of 48.8 months (range 1-82 months).

33 patients $(78.4 \%)$ were discharged postoperatively from hospital without any complication. Postoperative complications were

(1) there was post-CPR loss of vision in ICU in one patient (after cardiac arrest);

(2) there was heart block in two cases, a complete and a partial one which were managed with and without pacemaker, respectively;

(3) because of huge tumor size and chronic illness one patient experienced congestive heart failure;

(4) secondary to tumor emboli two patients developed cerebral complications (hemiplegia and aphasia);

(5) in one patient that postembolic coma was developed having many late complications including low LOC, prolonged mechanical ventilation, tracheotomy, blue toe, and bed sore; 
TABle 4: Patients' well-being in discharge time diagnosed with myxoma from 1994 to 2014 in Shahid Modarres Hospital.

\begin{tabular}{lc}
\hline Secle & $1994-2014$ \\
\hline No secle & $33(78.4 \%)$ \\
Blue toe + CVA + tracheostomy & $1(2.4 \%)$ \\
Right hemiparesia & $1(2.4 \%)$ \\
Right hemiparesia with aphasia & $1(2.4 \%)$ \\
Visual loss & $1(2.4 \%)$ \\
Heart failure & $1(2.4 \%)$ \\
Complete heart block & $1(2.4 \%)$ \\
PAT with heart block & $1(2.4 \%)$ \\
Discharge and death after 1 month & $1(2.4 \%)$ \\
Hospital mortality & $1(2.4 \%)$ \\
\hline Total & 42 \\
\hline
\end{tabular}

(6) one month after discharging from hospital one patient died due to noncardiac problems (DIC, hepatorenal syndrome and loss of consciousness);

(7) Preoperative CVA and pneumonia resulted in postoperative death in one case (Table 4).

Discharging from hospital 33 patients (78.4\%) had NSR (normal sinus rhythm). On the other hand, some others suffered from different dysrhythmia. AF: 4 patients, partial heart block: 3 patients, PAT with heart block: 1 patient and one patient had complete heart block.

Mean tumors' size was $5.22 \pm 1.6 \mathrm{~cm}$ (range of 2.2 to $8.2 \mathrm{~cm})$.

\section{Discussion}

Cardiac myxoma is the most important cardiac tumor. Its annual incidence is 5 in one million people. Each cardiac surgery center encounters one to two of this rare disease in year [3].

There were 42 cardiac myxomas in our hospital in last 20 years (incidence rate: 2.1 per year). Meanwhile in Middle East no precise statistic records about this tumor prevalence are at hand. However, there is a report of 35 cases in 5-year period (7 cases in each year) in Tabriz [4], which may be attributed to single referral cardiac surgery center in that city.

Zheng et al. reported 66 cardiac myxoma cases within 26 years in China (2.5 cases per year) [5]. One study reported 41 cases in 19 years in Greece ( 0.48 cases per year) [6]. Another report from Australia stated 24 cases in 30 years ( 0.8 cases per year) [7]. So far, the largest reported series is attributed to Xue et al. from China, posing 211 myxoma tumors in 18 years (11.72 cases per year) [8].

All referral literatures emphasize female predominance $[3,8]$ and in our study there was $1.47 \mathrm{female} / \mathrm{male}$ ratio. Also, this ratio was reported as 1.33 in Shahid Madani Hospital in Tabriz [9]; in contrast with this recent trend in Greece and China $[6,8]$ female predominance has been changed to male predominance (male/female rate in Greece: $26 / 15$ and in China 48/18).
These recent trends show a change in gender predominance which necessitates further study. According to many literatures disease clinical manifestations are evident in $3 \mathrm{rd}$ to 6 th decades of life $[3,10]$. In our study presentations were mostly reported in the same period (mean patients' age: 50.62 years). In Tabriz it was 52 years [9] and it has been mentioned to be 45 to 54 years in many other countries $[5,6,10]$. The youngest case in our study was 13 and oldest one was 76 . Kirklin believed myxoma has not been seen and reported in infancy but Pasaoglu reported one myxoma case in a 35-dayold infant in Turkey [11]. Also, there are reports of a 3-year-old child with cardiac myxoma by Zheng et al. [5] and another 4year-old child by Rebollar et al. [12].

In our study mean time from diagnosis to operation was 5.83 days (range of 1 to 48 days) and most operations were performed in one or two days after diagnosis.

Since the disease is naturally lethal and it requires urgent cardiac operation, there was no report of waiting time to operation in most cardiac surgical centers $(8-10 \%$ of patients die while waiting for operation) $[3,5]$.

Common causes of delay in operation are patients' fear of operation, waiting for more diagnostic modalities such as coronary angiography, CT scan and TEE, and finally long waiting list in public hospitals [13]. In our study, mean hospital stay was 12.7 days (range of 5 to 32 days) which is comparable to reported results of one study with mean ICU stay of $2.3 \pm 0.8$ days and mean hospital stay of $7.9 \pm 1.8$ days [5].

Most common clinical symptoms in this study were dyspnea, dyspnea with palpitation and chest pain which were similar to other studies [3-6]. Although rate of embolic events has been reported to be about $5 \%$ in most literatures, in our study this rate was higher (7.1\%). In Tabriz study, embolic events rate was $8.6 \%$ and $2.4 \%$ in Samanidis study $[6,9]$ for which we cannot explain the reason of differences in embolic events rate. It is not clear whether all patients with low level of consciousness are evaluated for cardiac tumors or all embolectomy specimens are sent for pathologic evaluation.

In all patients primary diagnostic modality was TTE. For 5 of them additionally TEE was conducted as well. Depending on patient's age, coronary angiography was applied to evaluate atherosclerosis (in 19 patients) leading to one concurrent CABG and myxoma operation. Although TEE can diagnose small tumors of 1-3 mm diameter, waiting for TEE can delay operation up to two weeks. So, it is not clear whether delay for TEE is beneficial for patient or not; also it is not certain if this delay favors or changes operation method.

Except one recurrent case $(2.4 \%)$ who had a familial cancer history all myxomas were primary. Nearly $5 \%$ of myxomas are familial (autosomal dominant), they are mainly younger patients, and there is not gender predominance in this group $[3,10]$.

In $20 \%$ of them (familial group), tumor originates from atrium and ventricle. This group have abnormal DNA chromosomal genotype pattern $[3,6,8]$. In most literatures, tumors were found in LA (75\%) and in RA (10-20\%) and in ventricles in remaining cases $(3)[3-5,8,11]$. In our study tumors were found in LA (85.6\%) and in both atria $(4.8 \%)$ and in RA (2.4\%). Mean tumor diameter was $5.22 \mathrm{~cm}$ which 
is similar to that of other literatures (mean diameter of 5$6 \mathrm{~cm})[3,10]$. In the study of China mean tumor diameter was about $0.3-2.5 \mathrm{~cm}$ and in the study of Greece it was about $5.1 \mathrm{~cm}[5,6]$.

There is no general agreement about repairing septal defect after tumor resection. Many surgeons repair it with artificial patches such as Dacron $[5,12,14,15]$ and others repair it with pericardial patch.

We prefer to repair septal defect with pericardial patch. In our center, septal defects were repaired with pericardial patch in $42.8 \%$ and with primary approach in $52.4 \%$ of cases. In one case with myxoma located on ventricular septum tumor was excised with partial septal resection [16].

In some occasions other cardiac operations are concurrently essential. We experienced a concurrent CABG, one pulmonary valve repair, two mitral valve repairs, and two mitral valve replacements. There are reports of concurrent CABG [17] and CABG with MVR in addition to myxoma removal [5].

Like other literatures, we witnessed postoperative arrhythmia after septal removal (21.42\%) [3, 5, 13].

While average hospital mortality is around 5\% $[3,5,10]$ we observed that as $2.4 \%$, one case with preoperative critical general condition (the patient was admitted in hospital with CVA and then developed pneumonia). Recent studies have reported near zero mortality $[5,6]$ which may be contributed to access to equipped referral hospital.

\section{Conclusion}

Myxoma tumors' prevalence in Iran was the same as that in other countries in the Middle East; however, its rate is less than that in East of Asia and more than that in Europe. These recent results indicate a change gender predominance, mean time from diagnosis to operation was too long. The patients had more preoperative embolic events and preoperative complications. In terms of size and location it was approximately parallel to other literatures.

AS vague chest pain and remote embolic events can raise doubt about myxoma existence. We recommended that in all patients with these findings myxoma should be ruled out.

\section{Consent}

Written informed consent was obtained from all participants for this paper and its accompanying images. A copy of the written consent is also available for review.

\section{Conflict of Interests}

The authors declare that they have no competing interests.

\section{Authors' Contribution}

All authors contributed extensively to the work presented in this paper. Dr. Hamid Ghaderi was a major contributor in writing the paper, prepared the paper and wrote the discussion, and was the corresponding author and helped in all aspects. Dr. Zahra Ansari Aval and Dr. Mahnoosh Foroughi were members of surgical team and they helped in data collection as well. Dr. Seyedeh Adeleh Mirjafari helped in study concept and design and was a major contributor in the literature search and preparing the discussion. Dr. Hassan Tatari was in charge of drafting of the paper. Dr. Isa Khaheshi and Kamal Fani did critical revision of the paper for important intellectual content. Dr. Mohammad Forozeshfard was statistics analyst. All authors contributed to the writing of the paper and they read and approved the final paper.

\section{References}

[1] G. Lamba and W. H. Frishman, "Cardiac and pericardial tumors," Cardiology in Review, vol. 20, no. 5, pp. 237-252, 2012.

[2] I. Tasoglu, U. Tutun, G. Lafci et al., "Primary cardiac myxomas: clinical experience and surgical results in 67 patients," Journal of Cardiac Surgery, vol. 24, no. 3, pp. 256-259, 2009.

[3] L. H. Cohn, Cardiac Surgery in the Adult, McGraw-Hill Professional, New York, NY, USA, 4th edition, 2012.

[4] R. Safiarian and M. Hekmat, "Ten year evaluation of cardiac myxoma surgical approaches in Tehran Shahid Modarres Hospital during 1988-1997," Hakim, vol. 2, no. 4, pp. 286-293, 1999.

[5] J. J. Zheng, X. G. Geng, H. C. Wang, Y. Yan, and H. Y. Wang, "Clinical and histopathological analysis of 66 cases with cardiac myxoma," Asian Pacific Journal of Cancer Prevention, vol. 14, no. 3, pp. 1743-1746, 2013.

[6] G. Samanidis, K. Perreas, P. Kalogris et al., "Surgical treatment of primary intracardiac myxoma: 19 years of experience," Interactive Cardiovascular and Thoracic Surgery, vol. 13, no. 6, pp. 597-600, 2011.

[7] J. A. Smith, B. B. Davis, G. R. Stirling et al., "Clinicopathological correlates of cardiac myxomas: a 30-year experience," Cardiovascular Surgery, vol. 1, no. 4, pp. 399-402, 1993.

[8] G. Xue, Q. Wu, and F. Lu, "Some new concepts of cardiac myxoma," Zhonghua Wai Ke Za Zhi, vol. 33, no. 6, pp. 323-325, 1995.

[9] N. Safaei, N. Maghamipour, and E. Karimian, "Surgical experience with intracardiac myxomas," Acta Medica Iranica, vol. 45, no. 5, pp. 369-372, 2007.

[10] K. B. H. Kirklin, Ed., Kirklin/Barratt-Boyes Cardiac Surgery, Elsevier Saunders, Philadelphia, Pa, USA, 2013.

[11] I. Pasaoglu, M. Demircin, S. Ozkutlu, and A. Y. Bozer, "Righ atrial myxoma in an infant," Japanese Heart Journal, vol. 32, no. 2, pp. 263-266, 1991.

[12] A. C. Rebollar, E. O. Ramírez, L. P. Garza, F. J. de la Fuente Magallanes, H. R. González, and R. A. Cárdenas, "The surgical treatment of cardiac myxomas: 10 years of experience," Archivos del Instituto de Cardiologia de Mexico, vol. 62, no. 2, pp. 121-126, 1992.

[13] S. M. M. Tehrani, H. Ghaderi, M. Foroughi et al., "Ten-year evaluation of cardiac myxoma in Shahid Modarres hospital," Tehran University Medical Journal, vol. 72, no. 2, pp. 87-95, 2014.

[14] B. P. C. V. Paelinck, P. H. M. J. Vermeersch, C. G. Convens, P. A. van Cauwelaert, and l. F. J. van Den Branden, "Cardiac myxoma in thirteen patients," Nederlands Tijdschrift voor Geneeskunde, vol. 139, no. 38, pp. 1931-1935, 1995.

[15] S. Premaratne, N. W. Hasaniya, H. Y. Arakaki, M. M. Mugiishi, R. T. Mamiya, and J. J. McNamara, "Atrial myxomas: experiences with 35 patients in Hawaii," The American Journal of Surgery, vol. 169, no. 6, pp. 600-603, 1995. 
[16] M. Hekmat, S. Rafieian, Z. A. Aval, M. Khani, M. Taherkhani, and M. R. Movahed, "Asymptomatic pediatric pulmonic valve myxoma involving the right ventricular outflow tract: a case report and review of the literature," Journal of Heart Valve Disease, vol. 21, no. 3, pp. 398-400, 2012.

[17] S. Braun, H. Schrötter, K. Reynen, C. Schwencke, and R. H. Strasser, "Myocardial infarction as complication of left atrial myxoma," International Journal of Cardiology, vol. 101, no. 1, pp. 115-121, 2005. 


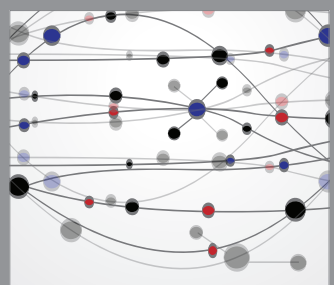

The Scientific World Journal
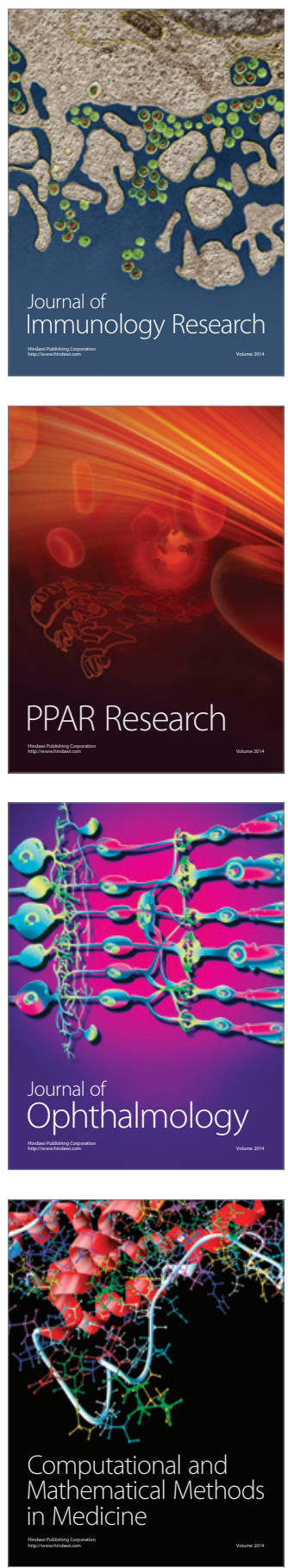

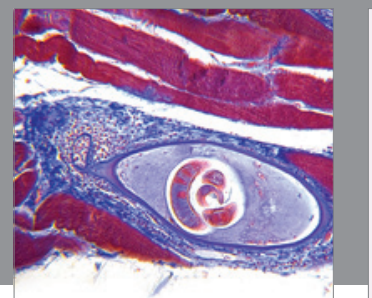

Gastroenterology

Research and Practice
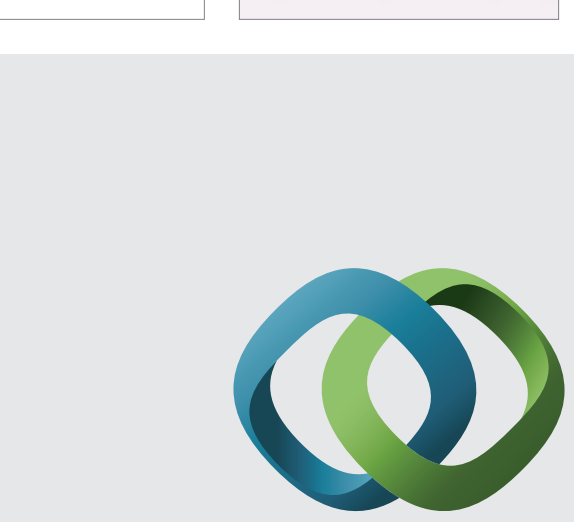

\section{Hindawi}

Submit your manuscripts at

http://www.hindawi.com
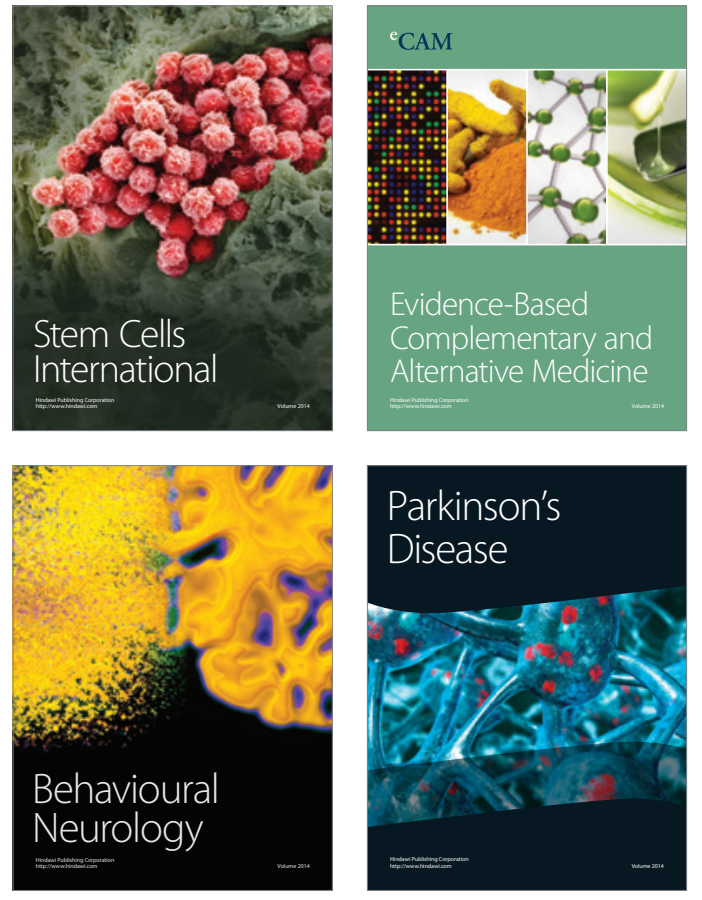
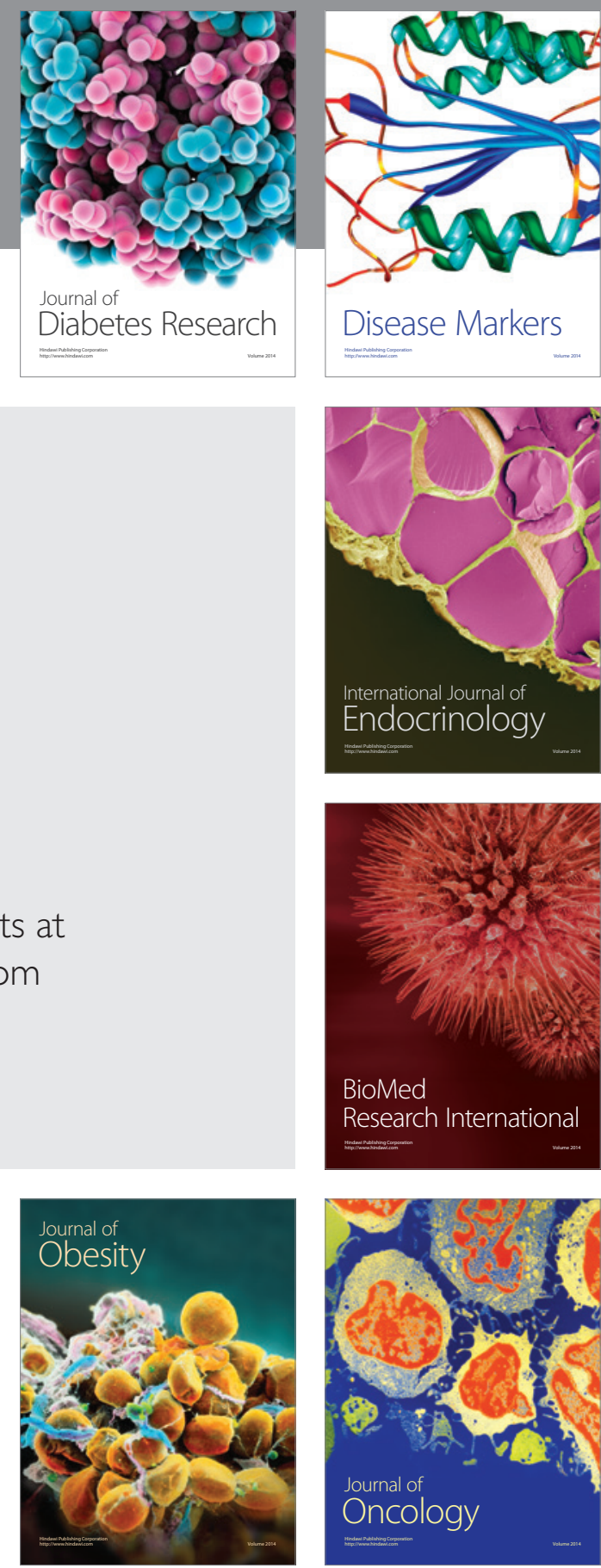

Disease Markers
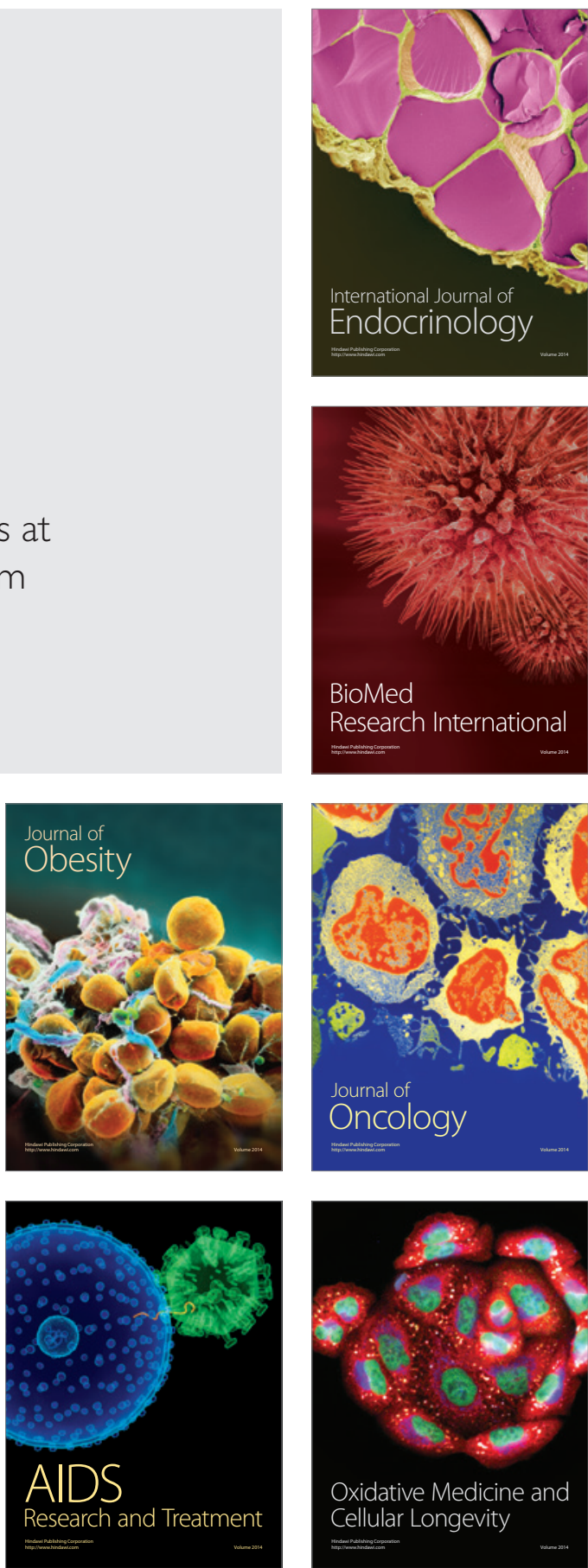